\title{
Kinematics and Control of Frog Hindlimb Movements
}

\author{
DAVID J. OSTRY, ANATOL G. FELDMAN, AND J. RANDALL FLANAGAN \\ McGill University, Montreal, Canada; and Institute for Information Transmission Problems, \\ Academy of Sciences, Moscow, USSR
}

\section{SUMMARY AND CONCLUSIONS}

1. The determinants of the motion path of the hindlimb were explored in both intact and spinal frogs. In the spinal preparations the kinematic properties of withdrawal and crossed-extension reflexes were studied. In the intact frog the kinematics of withdrawal and swimming movements were examined. Frog hindlimb paths were described in joint angle (intrinsic) coordinates rather than limb endpoint (extrinsic) coordinates.

2. To study withdrawal and crossed-extension reflexes, the initial angles at the hip, knee, and ankle were varied. Withdrawal and crossed extension were recorded in three dimension (3-D) with the use of an infra-red spatial imaging system. Swimming movements against currents of different speeds were obtained with high-speed film.

3. Three strategies were considered related to the form of the hypothesized equilibrium paths specified by the nervous system: all trajectories lie on a single line in angular coordinates; all trajectories are directed toward a common final position; and all trajectories have the same direction independent of initial joint configuration.

4. Joint space paths in withdrawal were found to be straight and parallel independent of the initial joint configuration. The hip and knee were found to start simultaneously and in $75 \%$ of the conditions tested to reach maximum velocity simultaneously. Hip-knee maximum velocity ratios were similar in magnitude over differences in initial joint angles. This is consistent with the observation of parallel paths and supports the view that the nervous system specifies a single direction for equilibrium trajectories.

5. Straight line paths with slopes similar to those observed in withdrawal in the spinal preparation were found in swimming movements in the intact frog. Straight line paths in joint space are consistent with the idca that swimming and withdrawal are organized and controlled in a joint-level coordinate system. The similarities observed between spinal and intact preparations suggest that a common set of constructive elements underlies these behaviors.

6. Path curvature was introduced when joint limits were approached toward the end of the movement. Depending on the initial joint angles, the joint movements ended at different times. When initial joint angles were unequal, joints moving from smaller initial angles reached their functional limits earlier and stopped first.

7. In withdrawal and crossed extension in the spinal frog, velocity profiles at a given joint were similar over the initial portion of the curve for movements of different amplitude. This is consistent with the idea that withdrawal and crossed-extension movements of different amplitude are produced by a constant rate of shift of the equilibrium position.

\section{INTRODUCTION}

Recent behavioral studies of spinal reflexes and sensorimotor organization in amphibians and reptiles have focused on the kinematics of reflex wiping and scratching (e.g., Berkinblit et al. 1986; Giszter et al. 1989; Mortin et al. 1985). In this paper we have examined the kinematics of flexion and crossed-extension reflexes (cf. Schotland et al. 1989). These reflexes were tested to assess the form of the control signals subserving hindlimb movement in the frog and the role of segmental mechanisms in multijoint coordination.

The kinematics of hindlimb withdrawal and crossed-extension reflexes were examined in both intact and spinal frogs. Swimming movements in the intact preparation are also described. Swimming cannot be elicited in the spinal frog, and thus the role of spinal mechanisms in this behavior cannot be directly assessed. Nonetheless, it is important to explore whether properties of spinal function in reflex behaviors are present in behaviors that require supraspinal input.

We have experimentally manipulated the initial joint angles associated with the hindlimb withdrawal and crossedextension reflexes to determine the effects of initial joint configuration on the form of the hindlimb trajectory. In Fig. 1, we display hindlimb motion paths in joint space based on three alternative movement strategies. The figure shows movements starting from different initial joint configurations. One possibility $(A)$ is that the nervous system produces trajectories that converge to a single line in joint space. Thus movements starting from different initial joint configurations would all approach a common path. A second alternative $(B)$ is that the system generates straight line trajectories toward a common final configuration. In this case we would observe nonparallel straight line paths in joint coordinates. A third alternative $(C)$ is that the system produces trajectories with a common direction. This would result in parallel motion paths (until joint limits are approached) in which the ratio of joint velocities over time is preserved in spite of different initial joint configurations.

These alternatives have been distinguished on the basis of kinematics. However, they can also be related to the equilibrium trajectories (times sequences of equilibrium positions), which we assume underlie the generation of motions. Care must be taken in relating equilibrium trajectories to actual trajectories. The deviation between them will depend on the form of the equilibrium trajectory (path and velocity profile), the initial limb configuration, and the movement dynamics. In alternative one, we suggest that the nervous system specifies a unique equilibrium trajectory that is insensitive to initial limb configuration. All actual trajectories will converge to this equilibrium trajectory. In alternative two, we assume that the system selects the direction of the equilibrium trajectory toward a common final configuration. Thus the equilibrium trajectory is sen- 


\section{Joint Space Paths}

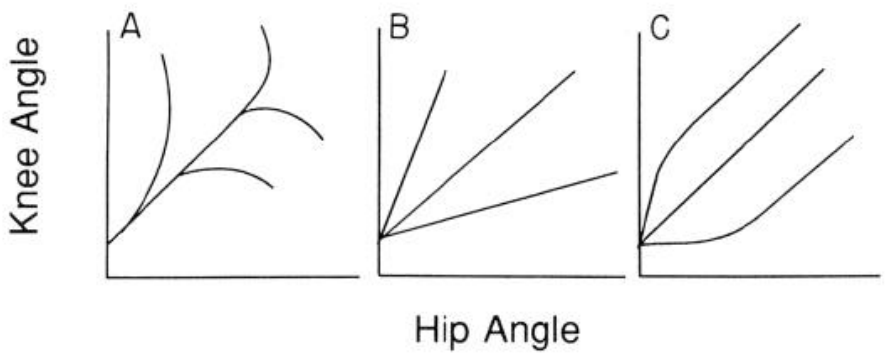

FIG. 1. Flexor reflex motion paths predicted by different movement strategies. $A$ : equilibrium positions lie on a single line in angular coordinates. $B$ : direction of equilibrium trajectory toward a final configuration is specified. $C$ : equilibrium trajectories all have a common direction.

sitive to initial joint angles. Finally, in alternative three, we suggest that the system specifies equilibrium trajectories with a single direction that does not depend on initial configuration. Changes in actual movement direction toward the end of the movement can be attributed to the joint reaching its limiting range rather than to changes in the direction of the equilibrium trajectory.

According to the equilibrium point (EP) hypothesis (see Feldman 1986; Feldman et al. 1990; Flanagan et al. 1989), kinematics arise as a consequence of shifts in equilibrium. Any difference between the current and equilibrium positions of the system gives rise to additional recruitment or derecruitment of motoneurons, which, in turn, results in muscle forces that restore equilibrium. Central commands control this process through the regulation of motoneuron excitability. Thus kinematics are only indirectly related to the central commands. Nevertheless, kinematics provide a window into the form of the underlying equilibrium trajectories. Although actual trajectories depend on limb dynamics, these effects are lessened in the behaviors studied here as a result of the relatively low hindlimb inertia. In addition, the opposing directions of link rotation tend to reduce dynamic effects because of interaction torques and coupling inertial torques. However, these effects are still present. For example, muscles that flex the hip will also tend to flex the knee because of inertial coupling. Thus, during withdrawal, mechanics would tend to produce synchronous hip and knee flexion.

\section{METHODS}

Hindlimb withdrawal and crossed-extension reflexes were recorded in the spinal frog. Withdrawal and swimming movements were recorded in intact frogs. Rana catesbeiana, Rana pipiens, and Rana temporaria were used in the study.

In total, large samples of withdrawal data were obtained from three $R$. temporaria and four $R$. catesbeiana. The $R$. temporaria were studied both before and after transection. Crossed-extension reflexes were recorded in two $R$. catesbeiana after transection. Swimming movements were obtained in two $R$. pipiens and one R. temporaria.

Withdrawal and crossed extension were recorded in three dimension (3-D) with the use of an infra-red spatial imaging system. Both behaviors were elicited by mechanical stimulation of hindlimb. Infra-red-emitting diodes (IREDs) were attached by glue or sutured to the skin along the midline of the back at the level of the ileosacral joint and cloacal fold as well as to the knee, ankle, and tarsal-metatarsal joints (see Fig. 2). Light-weight low-torsion wiring was used to reduce mechanical resistance to movement.

In studying the withdrawal reflex, the initial angles at the hip, knee, and ankle were varied systematically. Each joint was examined at 90 and $135^{\circ}$. All eight possible combinations of initial joint angles were tested ( 1 of the initial configurations is shown in Fig. 2). Between 10 and 20 observations per condition per frog were collected. To study the crossed-extension reflex, we varied the initial configurations of the stimulated (ipsilateral) and the extending (contralateral) limb as well as the actual stimulation site. In both withdrawal and crossed-extension recordings, the infra-red markers were sampled at $400 \mathrm{~Hz}$.

High-speed filming at $100 \mathrm{~Hz}$ was used to record the patterns of angular motion of the hip, knee, and ankle in swimming. An underwater treadmill enabled the examination of swimming at both slow and fast current flow rates. Swimming in an open tank was also recorded. Twenty to 30 swimming cycles per frog per condition were collected. Markers were glued to the skin along the midline of the back at the level of the shoulders, ileosacral joint, and cloaca as well as to the knee, ankle, and tarsal-metatarsal joints of

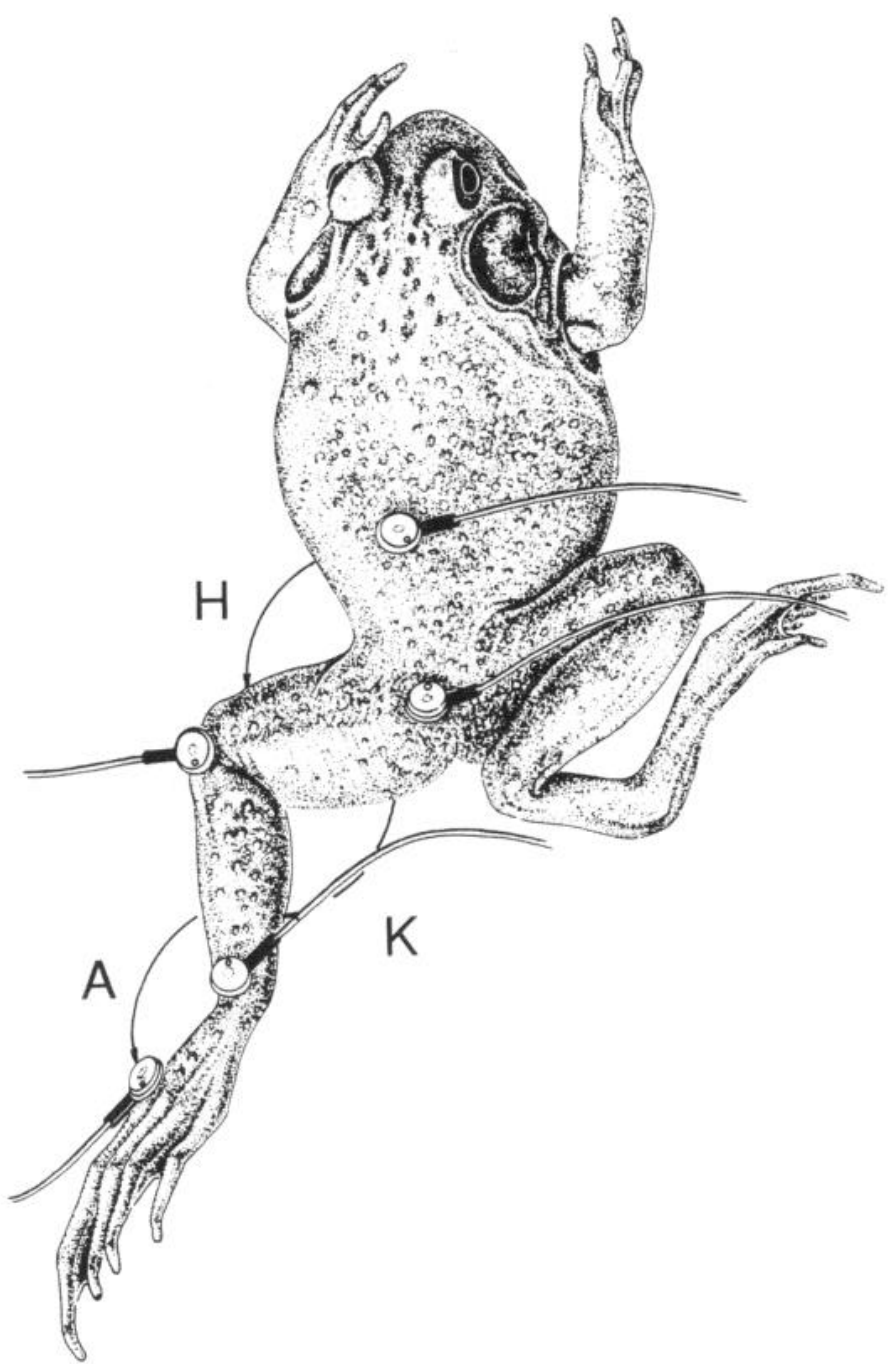

FIG. 2. Schematic drawing showing 1 of 8 initial joint configurations used in the study. Initial hip $(\mathrm{H})$ and knee $(\mathrm{K})$ angles of $90^{\circ}$ are combined with an initial ankle angle (A) of $135^{\circ}$. Locations of IREDs are indicated. 
both hindlimbs. The frog was filmed from above to record movements in a horizontal plane. Film records were digitized manually.

The trajectories of the individual markers were low-pass filtered with the use of a second-order zero phase lag Butterworth filter. The cut-off frequency was chosen on the basis of Fourier analysis and through direct comparison of raw and filtered records. In swimming, this frequency was between 6 and $10 \mathrm{~Hz}$ corresponding to a signal-to-noise ratio of $\sim 30 \mathrm{~dB}$. In the data recorded by the use of the infra-red imaging system, a low-pass frequency of 10 $\mathrm{Hz}$ was used; this corresponded to a signal-to-noise ratio $>40 \mathrm{~dB}$. Joint angles at the hip, knee, and ankle were computed by the use of the scalar product of the vectors defining the joints. Velocity and acceleration functions were derived by the use of the leastsquares method (Dahlquist and Björck 1969, Eq. 7.2.8)

$$
f_{i}^{\prime}=\left[2\left(f_{i+2}-f_{i-2}\right)+\left(f_{i+1}-f_{i-1}\right)\right] / 10
$$

where $f_{i}$ is the value of the function at time $i$, and $f_{i}^{\prime}$ is the first derivative at time $i$. When examining basic kinematic patterns, joint movement start and end times were determined on the basis of $10 \%$ of maximum joint angular velocity. However, in cases when exact start and end times were required (e.g., Figs. 13-15 in which the initial phases of joint velocity profiles are compared), movement start was taken as an absolute threshold angle of 10 $\mathrm{deg} / \mathrm{s}$.

Spinal transection was carried out under either tricaine anesthetic $(0.125 \%$, neutralized to $\mathrm{pH} 7)$ (see Ohr 1976 for details) or ether and verified visually with light microscope. Testing typically began on the third or fourth day after transection. All transections were carried out just caudal to the fourth ventricle. Postmortem verification was carried out by visual inspection of the spinal cord.

\section{RESULTS}

In this section we present movement paths in joint space as well as joint angular velocity profiles. In addition, a basic description of the amplitude and duration of joint motions is provided. We show that paths in joint space form straight lines, which start out parallel regardless of initial joint angles in withdrawal and current flow rate in swimming. It is also shown that, in withdrawal and crossed-extension reflexes, joint angular velocity profiles of movements of different amplitude at a single joint are similar over the initial portion of the movement. The results obtained for spinal and intact preparations are comparable.

In both swimming and withdrawal movements, we have focused on patterns of hip and knee motions. Ankle motion is not considered in detail because the duration and timing were variable. The pattern of hip and knee motion was found to be unaffected by the initial position, duration, and amplitude of the ankle. By focusing on the movements of the proximal joints, we are able to fully test the hypotheses, outlined in INTRODUCTION, related to the form of central control signals underlying hindlimb trajectory formation in the intact and spinal frog.

\section{Basic patterns of hindlimb movement}

Reflex withdrawal movements were elicited from eight different externally imposed initial joint angles. One goal was to assess the influence that movements at individual joints have on the kinematic patterns at other joints. We first examined this issue by determining the dependence of joint displacements, durations, and start and end times (staggering) on initial joint angles.
Figure 3 (top) shows typical position and velocity records for hindlimb withdrawal in spinal $R$. catesbeiana. The movement shown starts from an initial configuration in which hip, knee, and ankle angles are all $90^{\circ}$. The thin solid line represents the hip angle over time, the thick solid line represents the knee, and the dotted line the ankle. In general, movement amplitudes were greatest overall for the

\section{Withdrawal: Hip 90, Knee 90, Ankle 90}

\section{Position}
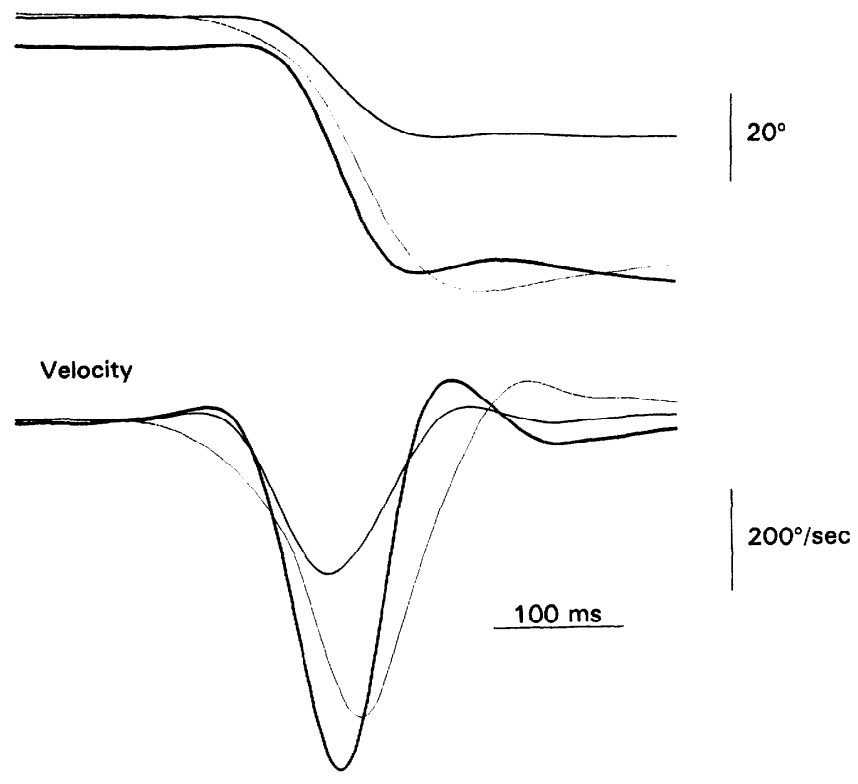

Crossed Extension
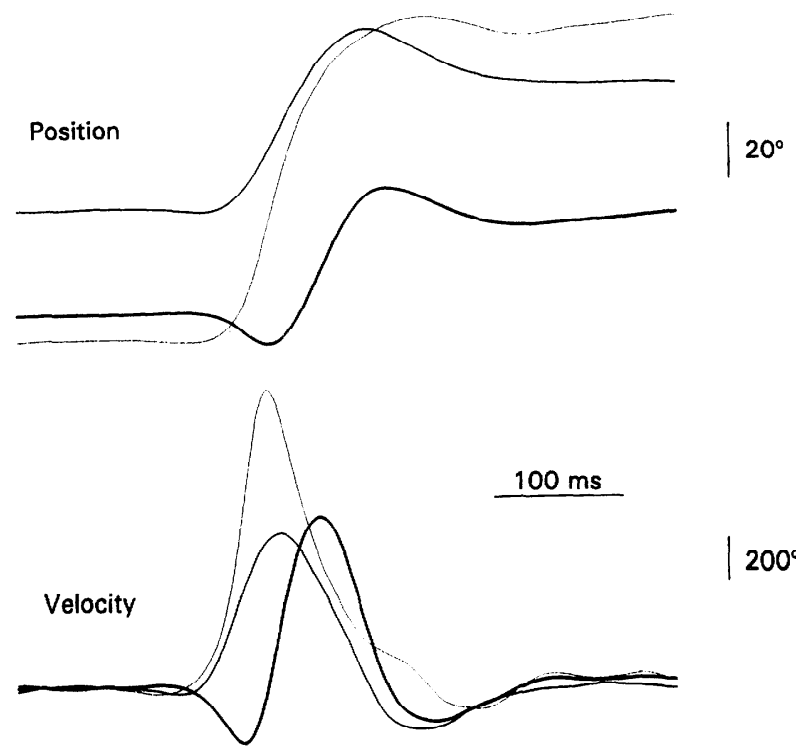

$200 \%$ sec

FIG. 3. Position and velocity records of flexion movement in withdrawal (top) and the extension movement in crossed extension (bottom) in the spinal frog. Hip, knee, and ankle are shown as thin, thick, and dotted lines, respectively. In withdrawal, the initial angles were all $90^{\circ}$. In the crossed-extension response, the stimulated limb was initially extended, and the contralateral limb was flexed. 
knee and least for the hip. Movement amplitudes and durations further depended on the initial angle at the joint (Figs. 4 and 5). This dependence of movement amplitude and duration on the initial joint configuration was assessed statistically, on a frog by frog basis, using analysis of variance (ANOVA).

The movement amplitudes of the hip, knee, and ankle were only dependent on the initial angle at the individual joint $(P<0.001)$ and did not depend on the initial angles of the other two joints. Movement amplitudes were always greater for larger initial joint angles. Similar patterns were observed for all frogs. (For FR2, the amplitude of knee and ankle movements also depended on the initial hip and knee angles, respectively; $P<0.0001$.)

The duration of the withdrawal reflex also depended on the initial joint angles. In all cases, the duration of hip movement was found to depend only on the initial angle at the hip $(P<0.01)$. Movement durations were longer for larger initial angles. Knee movements were likewise longer for larger initial angles $(P<0.001)$, except for frog $C H K$. Ankle movement durations did not vary systematically with either the initial ankle angle or the overall initial joint configuration.

The relationship between joint start and end times was examined for the hip and knee. Whereas hip and knee movements started at the samc time, their end times tended to differ. The order of movement ending depended on the initial angles at both joints. In general, joints with smaller initial angles stopped moving first. For example, when the initial angles at the hip and knee were 90 and $135^{\circ}$, respectively, movement of the knee ended after movement of the hip by $\sim 60-80 \mathrm{~ms}$ in three of the four frogs tested $(P<$ $0.01)$. In contrast, when the initial angles at the hip and knee were reversed, with hip angle initially $135^{\circ}$ and knee angle initially $90^{\circ}$, movement of the knee ended before movement of the hip by $\sim 30-60 \mathrm{~ms}(P<0.01$ for all frogs $)$.

\section{Average Movement Amplitude}

Withdrawal
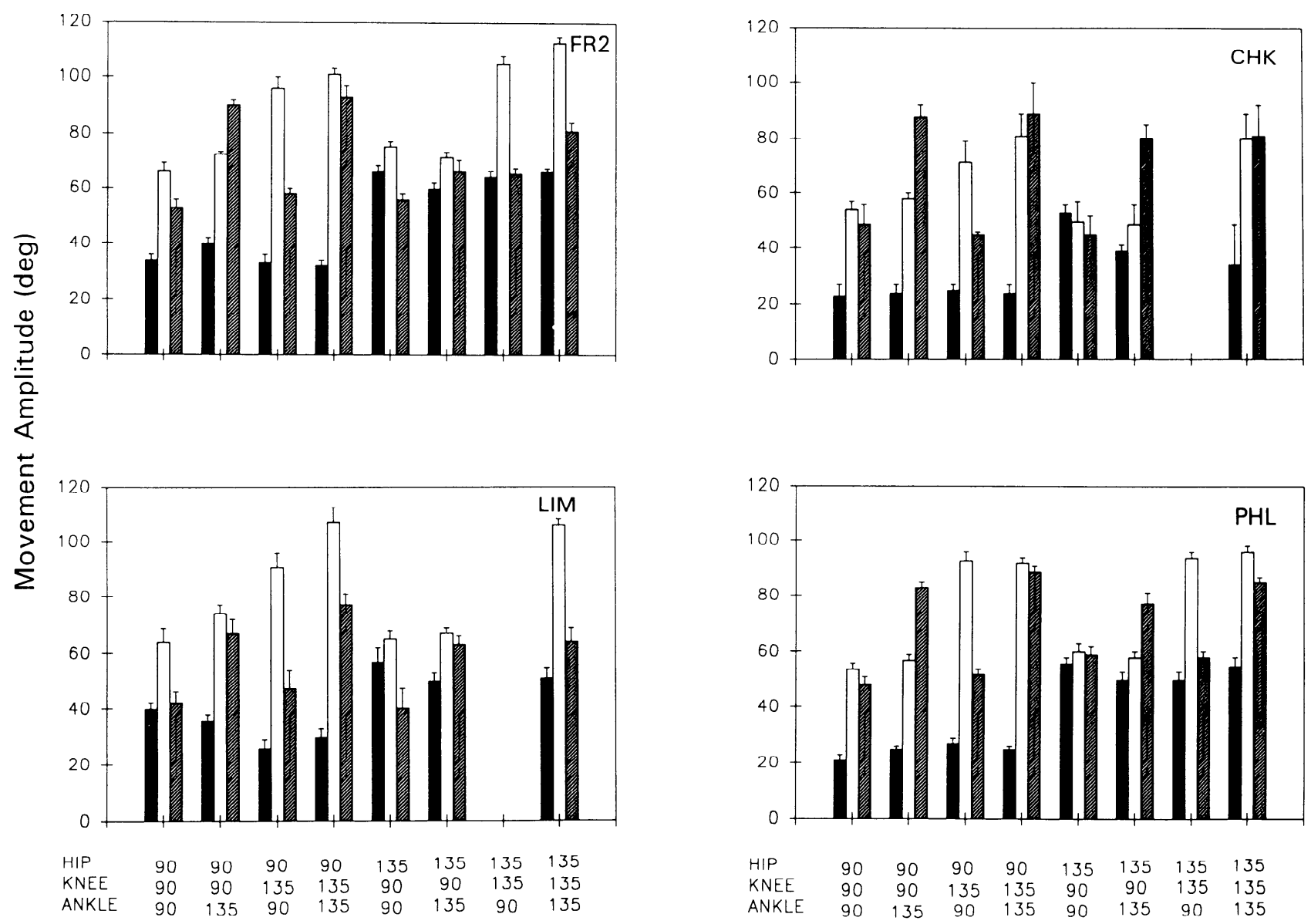

Initial Joint Configuration

FIG. 4. Average movement amplitude in degrees: hindlimb withdrawal response in spinal frog, (standard error in degrees). Note that the initial joint configuration involving 135,135 , and $90^{\circ}$ at the hip, knee, and ankle could not be achieved in frogs CHK and LIM. 


\section{Average Movement Duration}

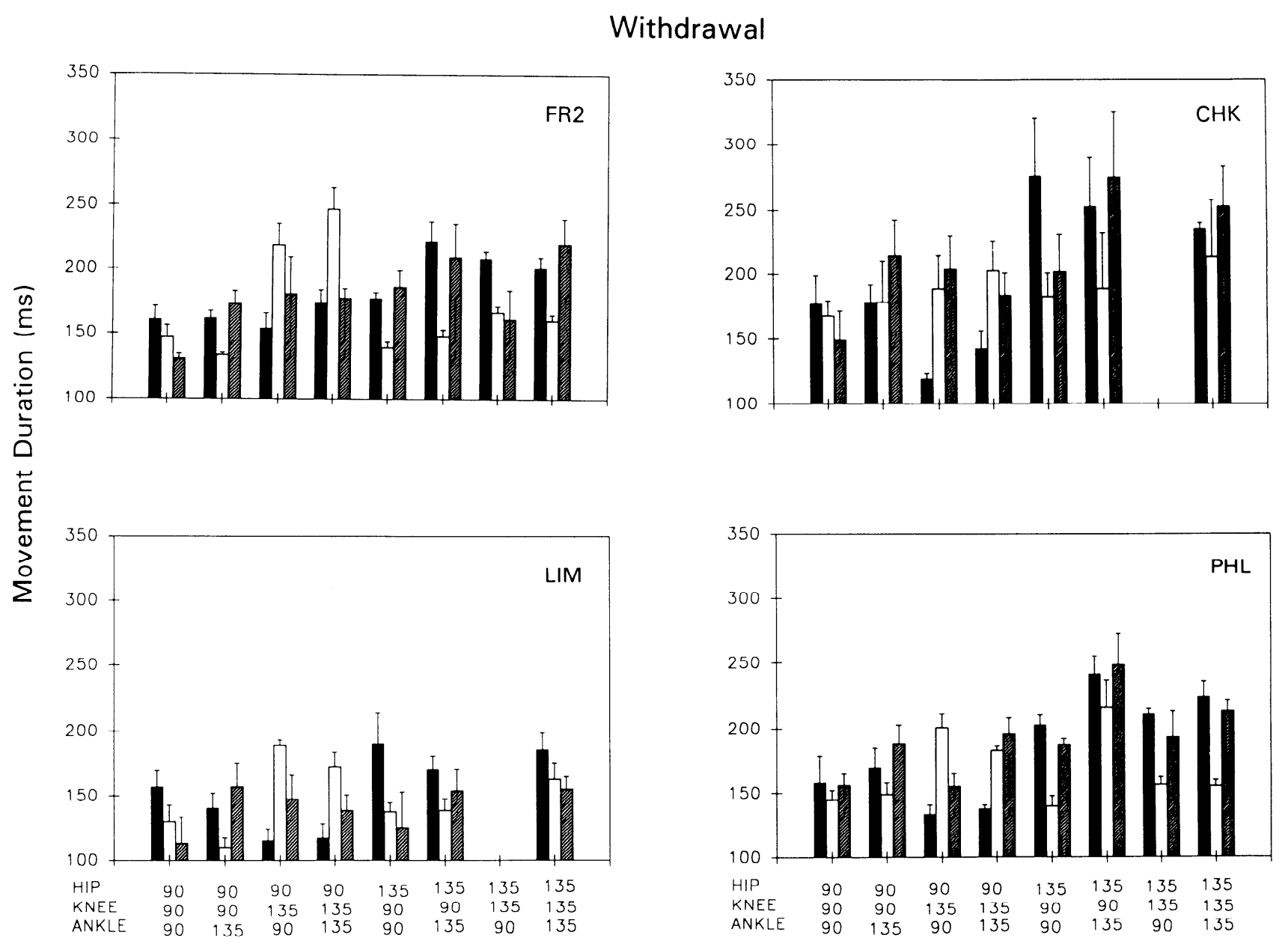

Initial Joint Configuration

FIG. 5. Average movement duration in milliseconds: hindlimb withdrawal response in spinal frog (standard error in milliseconds).

Thus both the amplitude and the duration of the hindlimb withdrawal movement in the spinal frog were greater for larger initial joint angles. The principle determinant of the amplitude and movement duration of a particular joint was the initial angle at that joint alone, independent of the angles at the other two joints. The start of hip and knee motions tended to be synchronous. The order of ending depended on initial angles. In particular, when initial joint angles were unequal, the joint whose initial angle was smallest ended moving first.

Swimming movements against imposed currents of different speeds were studied. Figure 6 (bottom) shows movements of both hindlimbs for swimming at a slow current rate. Hip, knee, and ankle angles are coded with thin, thick, and dotted lines, respectively. At slow current rates, an outof-phase interlimb pattern is observed. For swimming at a faster current rate, a characteristic in-phase interlimb pattern is obtained (Fig. 6, top). The dependence of interlimb coordination on speed is similar to that found in terrestrial locomotion in other species.
The swimming cycle comprises a movement component involving flexion followed by extension and may also include a rest component that is visible in in-phase kicking. Although the pattern of interlimb coordination used in swimming was affected by the current speed, neither the movement amplitude nor the duration of the movement component of the cycle varied with current speed. However, at both speeds, the duration and maximum velocity of the hindlimb flexion and extension movements differed reliably. Hindlimb extension movements, the propulsive phase of the cycle, had shorter durations $(P<0.01)$ and higher maximum velocities $(P<0.0001)$.

The average amplitudes and durations of the hindlimb flexions and extensions in swimming are given in Table 1. A comparison of these data with those for hindlimb withdrawal (Figs. 4 and 5) indicates that movement flexion durations were, on average, longer in swimming than flexion durations in withdrawal. Movement amplitudes in swimming were comparable to withdrawal movements involving large initial joint angles. However, even in these cases, 
Swimming: Fast Current

left

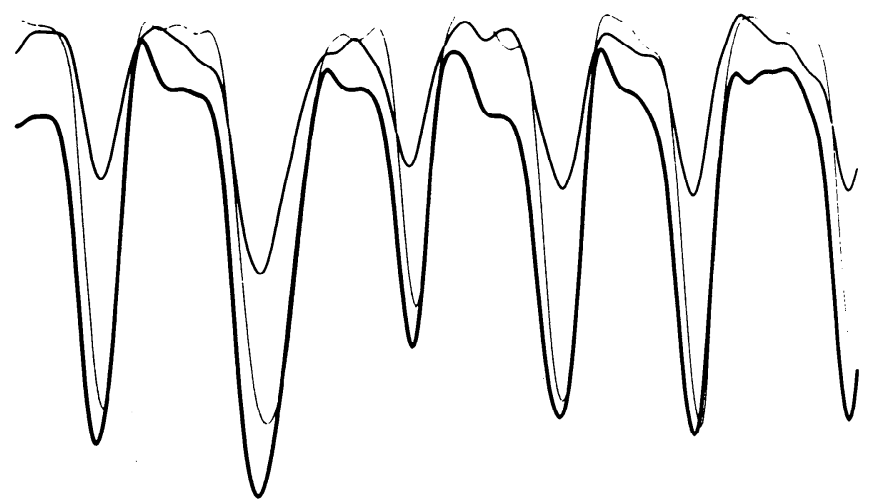

right

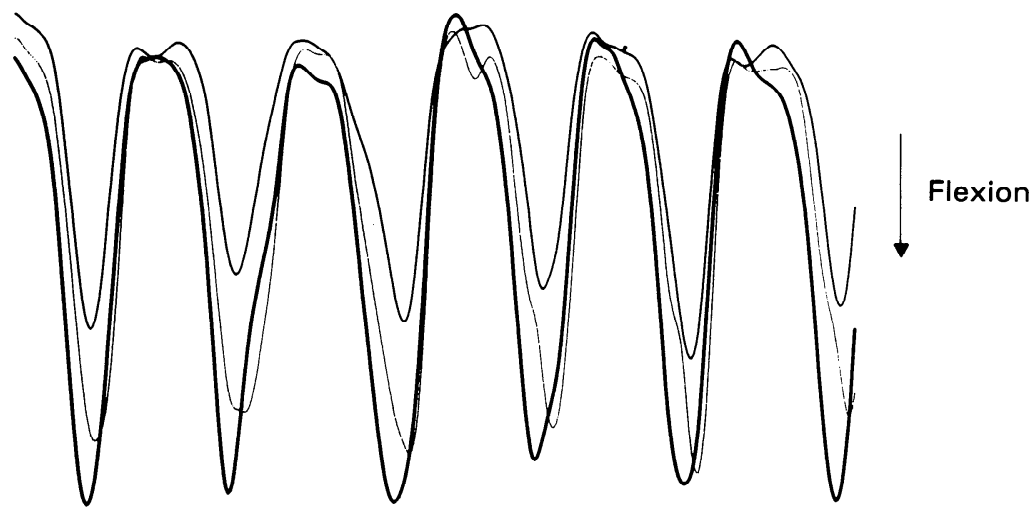

Swimming: Slow Current

left

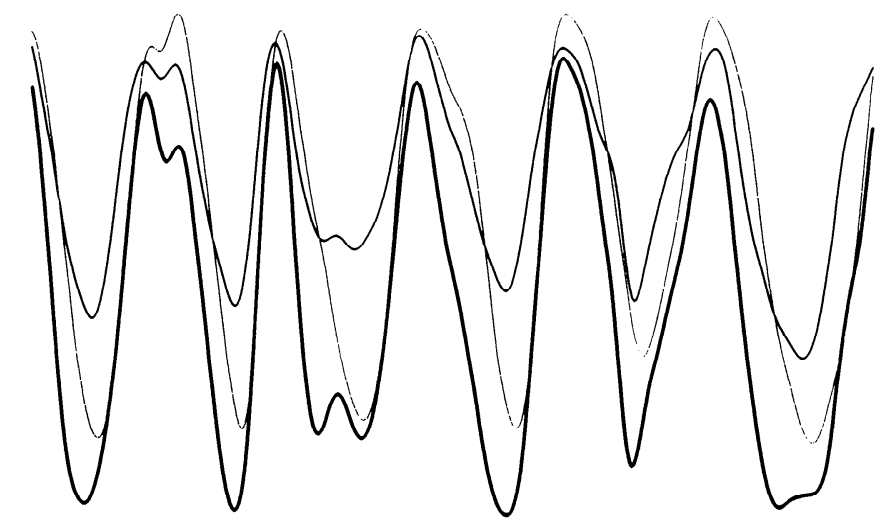

right

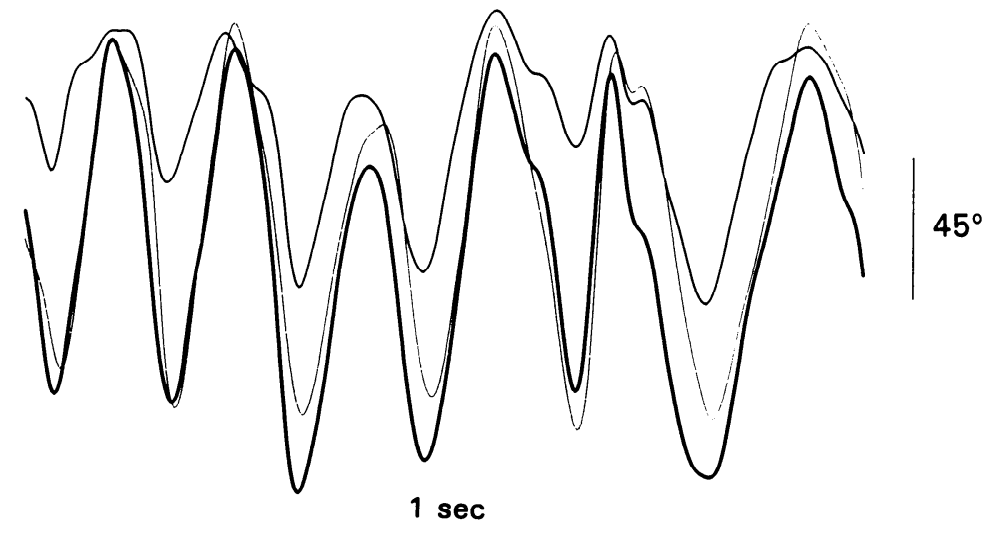

FIG. 6. Hindlimb swimming patterns in the intact frog. Hip, knee, and ankle are indicated by thin, thick, and dotted lines, respectively. Top: swimming movements of both hindlimbs at a fast current speed. Bottom: same frog swimming against a slow current. 
TABLE 1. Kinematics of hindlimb swimming movements in intact frog

\begin{tabular}{|c|c|c|c|c|c|c|c|c|}
\hline \multirow[b]{2}{*}{$\begin{array}{r}\text { Current speed } \\
\text { Movement }\end{array}$} & \multicolumn{4}{|c|}{ Average Amplitude, deg } & \multicolumn{4}{|c|}{ Average Duration, ms } \\
\hline & $\begin{array}{l}\text { Fast } \\
\text { flexion }\end{array}$ & $\begin{array}{l}\text { Slow } \\
\text { flexion }\end{array}$ & $\begin{array}{l}\text { Fast } \\
\text { extension }\end{array}$ & $\begin{array}{l}\text { Slow } \\
\text { extension }\end{array}$ & $\begin{array}{l}\text { Fast } \\
\text { flexion }\end{array}$ & $\begin{array}{l}\text { Slow } \\
\text { flexion }\end{array}$ & $\begin{array}{l}\text { Fast } \\
\text { extension }\end{array}$ & $\begin{array}{l}\text { Slow } \\
\text { extension }\end{array}$ \\
\hline \multicolumn{9}{|l|}{$\operatorname{Frog} A$} \\
\hline Hip & $66 \pm 4$ & $61 \pm 5$ & $67 \pm 4$ & $62 \pm 4$ & $287 \pm 28$ & $318 \pm 47$ & $249 \pm 12$ & $226+28$ \\
\hline Knee & $116 \pm 4$ & $111 \pm 7$ & $124 \pm 3$ & $113 \pm 7$ & $259 \pm 15$ & $365 \pm 39$ & $241 \pm 19$ & $241 \pm 34$ \\
\hline Ankle & $115 \pm 3$ & $106 \pm 5$ & $118 \pm 2$ & $109 \pm 5$ & $271 \pm 27$ & $278 \pm 36$ & $223 \pm 16$ & $221 \pm 22$ \\
\hline \multicolumn{9}{|l|}{ Frog B } \\
\hline Hip & $72 \pm 2$ & $70 \pm 6$ & $78 \perp 2$ & $75 \pm 5$ & $482 \pm 43$ & $420 \pm 29$ & $254 \pm 17$ & $373 \pm 78$ \\
\hline Knee & $120 \pm 3$ & $105 \pm 7$ & $129 \pm 2$ & $111 \pm 9$ & $589 \pm 71$ & $356 \pm 66$ & $293 \pm 28$ & $497 \pm 110$ \\
\hline Ankle & $108 \pm 2$ & $99 \pm 6$ & $114 \pm 3$ & $107 \pm 6$ & $442 \pm 35$ & $380 \pm 12$ & $241 \pm 15$ & $363 \pm 96$ \\
\hline \multicolumn{9}{|l|}{$\operatorname{Frog} C^{*}$} \\
\hline Hip & $71 \pm 3$ & & $79 \pm 3$ & & $411+25$ & & $236 \pm 11$ & \\
\hline Knee & $114 \pm 5$ & & $131 \pm 2$ & & $396 \pm 28$ & & $276 \pm 14$ & \\
\hline Ankle & $105 \pm 4$ & & $112 \pm 3$ & & $454 \pm 37$ & & $200 \pm 8$ & \\
\hline
\end{tabular}

Values are means $\pm \mathrm{SE} .{ }^{*}$ No current.

the duration of swimming movements was typically longer. As in the case of withdrawal, movements of the hip and knee typically began at the same time in swimming.

Kinematic data were obtained for the crossed extension in spinal $R$. catesbeiana. To study the crossed extension, we varied the initial configurations of the stimulated (ipsilateral) and the extending (or contralateral) limb as well as the actual stimulation site. In Fig. 3 (bottom) angular movements are shown of the hip, knee, and ankle in the crossedextension reflex. The movement of the contralateral or ex-

\section{Joint Space Paths}

\section{Withdrawal}
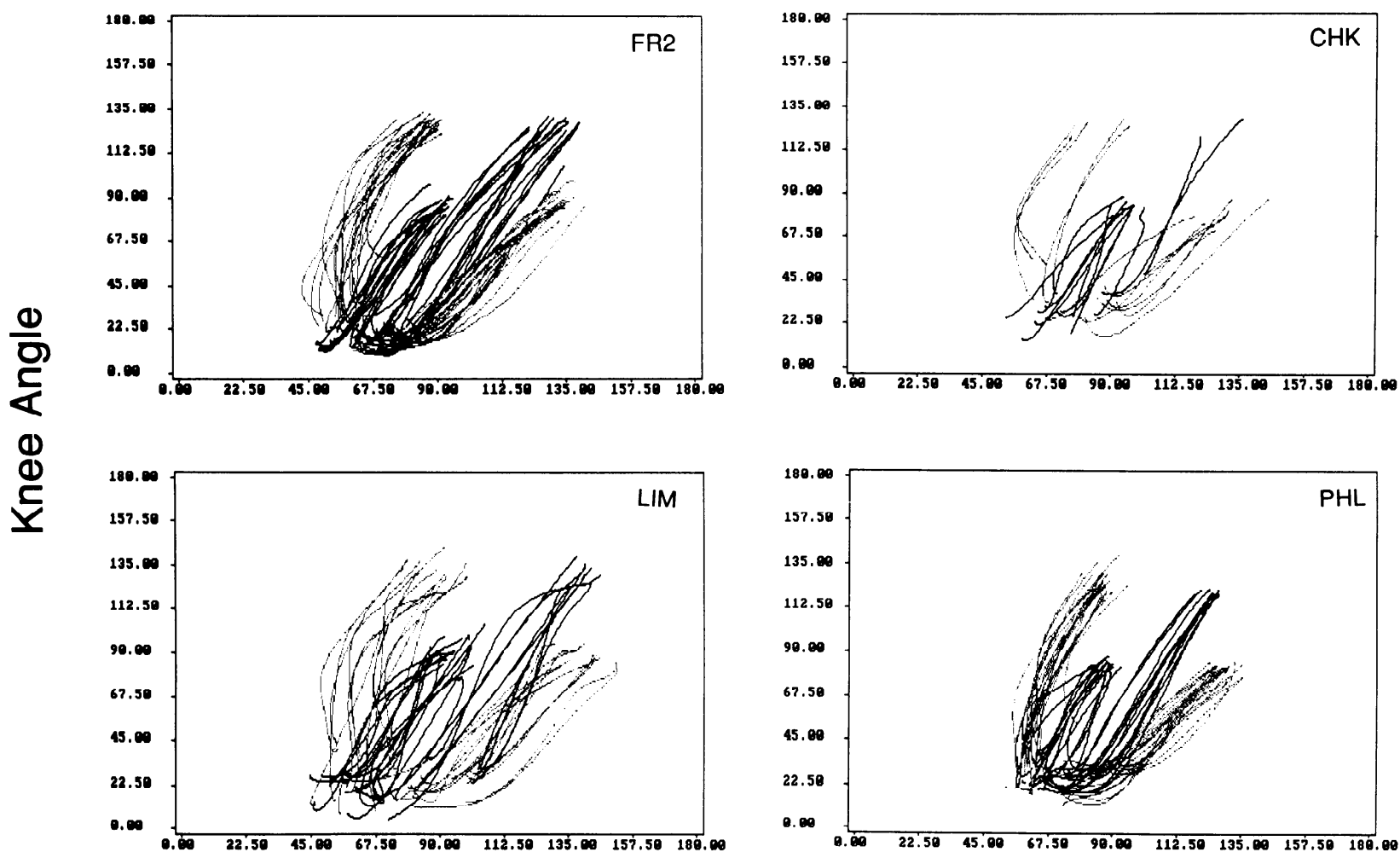

\section{Hip Angle}

FIG. 7. Hip angle vs. knee angle for withdrawal in the spinal frog. The movement path is from the top right to the bottom left of each panel. Paths for all initial joint configurations are shown. 
tending limb is presented. As can be seen in the velocity traces, typically the knee first flexes and then reverses direction and extends. Consequently, the extension phase of the knee movement lags the extension of the hip. In addition, the knee reaches maximum velocity after the hip. The pattern is different than flexion movements in withdrawal and swimming and, in particular, the extension movement in swimming where knee and hip motion begins simultaneously.

We also found that when the site of stimulation was changed from the ventral surface of the foot to the ventral surface of the metatarsus, which is the situation shown here,

\section{Joint Space Paths}

\section{Withdrawal}
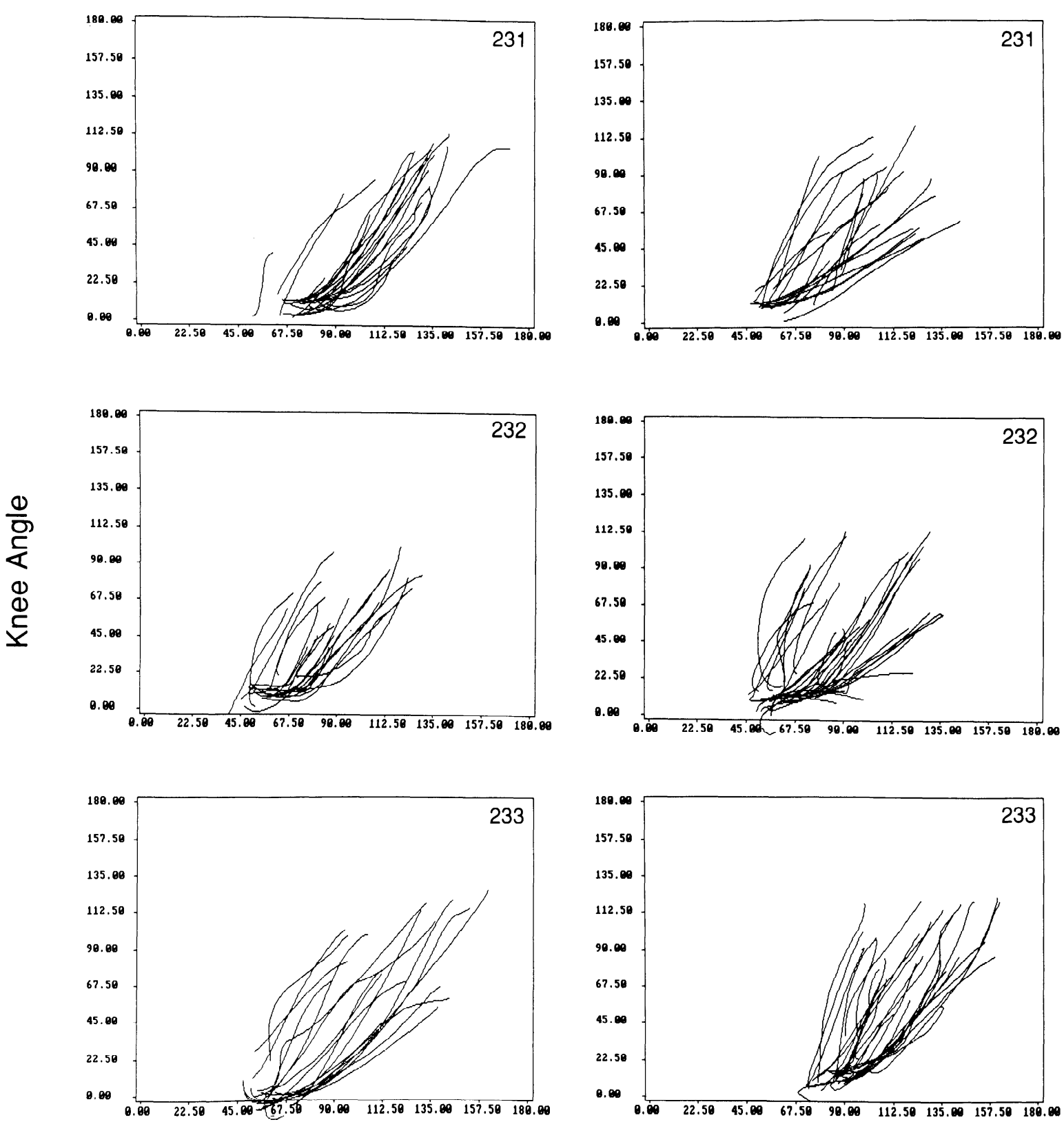

Intact 
the knee consistently flexed, at times as much as $20^{\circ}$, before its extension motion began. This effect did not depend on the initial configuration of the ipsilateral or the contralateral limb.

\section{Motion paths in joint space}

Figure 7 presents the observed paths in hip-knee space for the withdrawal reflex in the spinal frog. The hip angle is given on the horizontal axis, and the knee angle is on the vertical. The short paths in the center of the figure correspond to movements in which the initial hip and knee angles were both $90^{\circ}$, whereas longer paths to their immediate right are for movements in which hip and knee angles were both $135^{\circ}$. The traces in the top and bottom portions of each panel are for movements in which the initial angles at the hip and knee were unequal. Both of the initial joint configurations in which initial angles were equal can be seen to result in straight line paths, which are parallel, in hip-knee space. Note that the data in this figure were obtained from trials in which both initial ankle angles ( 90 and $135^{\circ}$ ) were tested. Thus the pattern of hip-knee coordination is unaffected by the initial ankle angle.

Parallel and straight line paths are not observed in hipknee space when the movement approaches the work-space boundary. Over the initial part of the movement, all paths are approximately parallel. (Note, however, that the initial slopes of the paths on the right of the figure are slightly less than the others.) In contrast, the traces tend to converge toward the end of the movement. We suggest that this is due to one of the joints approaching its functional limit before the movement end. Parallel and straight line paths with curvature toward the end of the movement are also observed in intact preparations. Figure 8 shows hip-knee paths for three $R$. temporaria both before and after spinal transection.

\section{Joint Space Paths}

\section{Movement Start to Maximum Velocity}
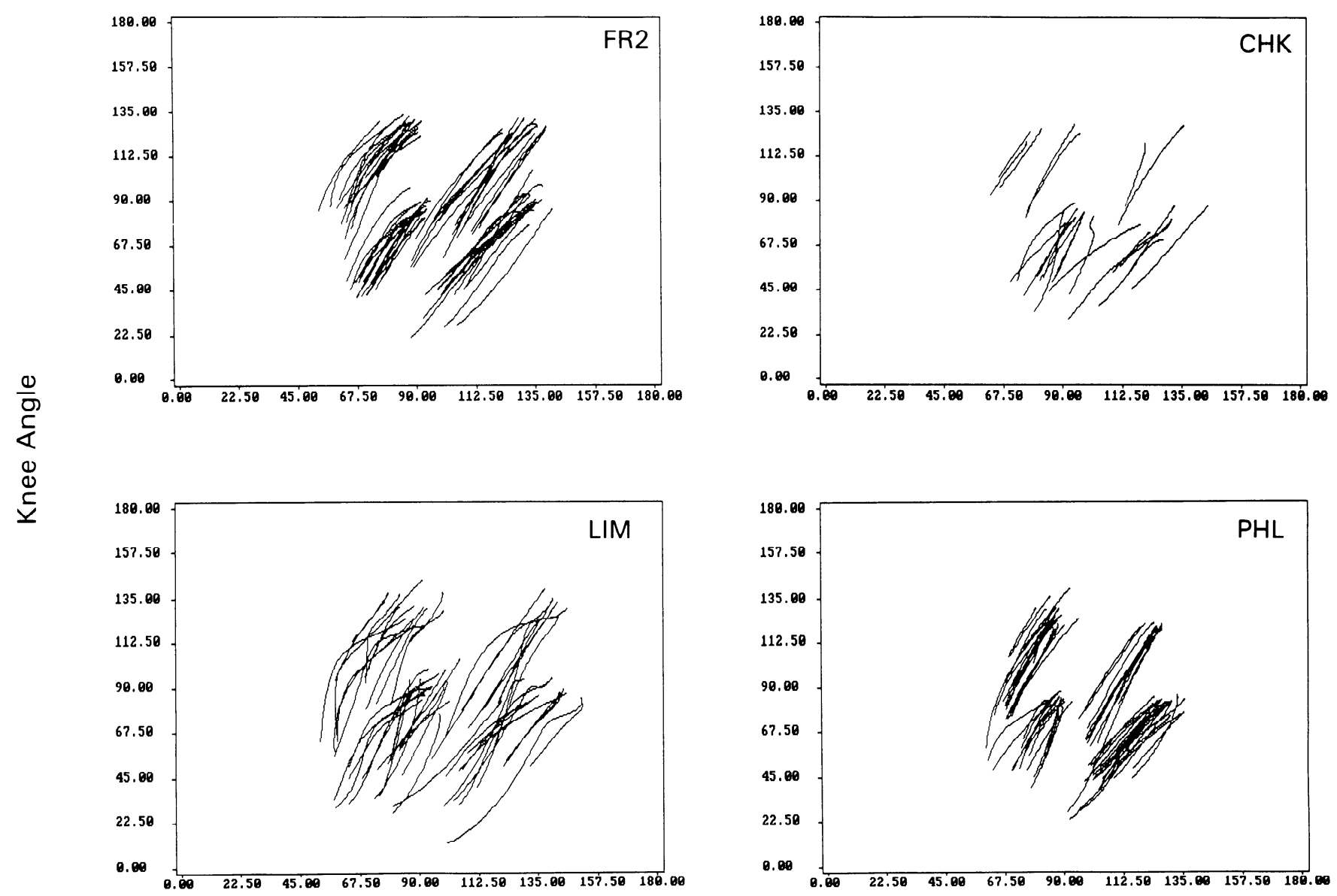

Hip Angle

FIG. 9. Hip-knee paths for withdrawal in spinal $R$. catesbeiana. Paths are shown from movement start until the time at which the 2 nd of the 2 joints reaches maximum velocity. 
The straight line paths in joint space indicate that the hip and knee start and end at the same time and the form of the hip and knee angular velocity functions is similar. Parallel paths indicate that the ratios of hip and knee velocities are constant regardless of initial configuration.

To provide quantitative support for the observation of parallel straight joint space paths, we calculated the ratio of maximum hip velocity to maximum knee velocity, the time difference between joint movement onsets, and the time difference between joint maximum velocities for each movement condition (i.e., initial joint configuration). If paths are parallel and straight, then the ratio of maximum velocities will not vary, the two joints will start moving at the same time, and the joints will reach maximum velocities at the same time. On the other hand, if the paths are straight but not parallel, then maximum velocities and joint onset times will occur simultaneously, but the ratio of maximum velocities will differ across conditions.

Figure 9 shows withdrawal reflex hip-knee paths from movement start to the time at which the second of the two joints reaches maximum velocity. In general, the paths from movement initiation to maximum velocity are straight and essentially parallel. This suggests that the joints start simultaneously, reach maximum velocity simultaneously, and that the ratio of maximum velocities is constant. When the initial combination of hip and knee angles is 90 and $135^{\circ}$, the paths for FR2, PHL, and LIM are curved. This suggests that maximum velocities in these conditions may not be reached simultaneously.

Differences in the movement start times and maximum velocity times of the hip and knee were evaluated in each condition separately by the use of $t$ tests. Overall, the aver-

\section{Hip - Knee Velocity Ratio}

\section{Withdrawal}
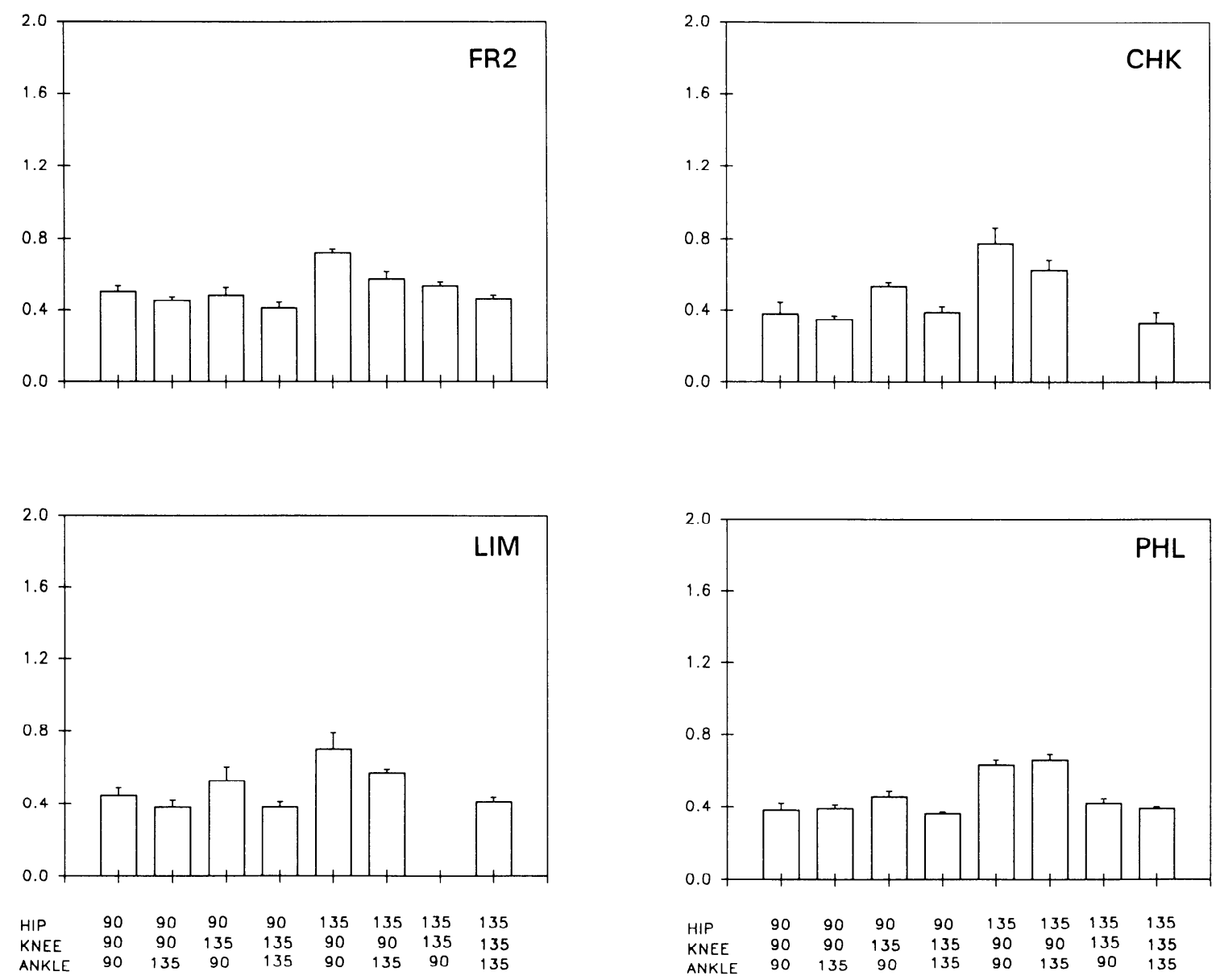

Initial Joint Configuration

FIG. 10. Ratios of maximum hip velocity to maximum knee velocity in withdrawal for spinal $R$. catesbeiana. Standard error bars are shown. 
Joint Space Paths

\section{Swimming}
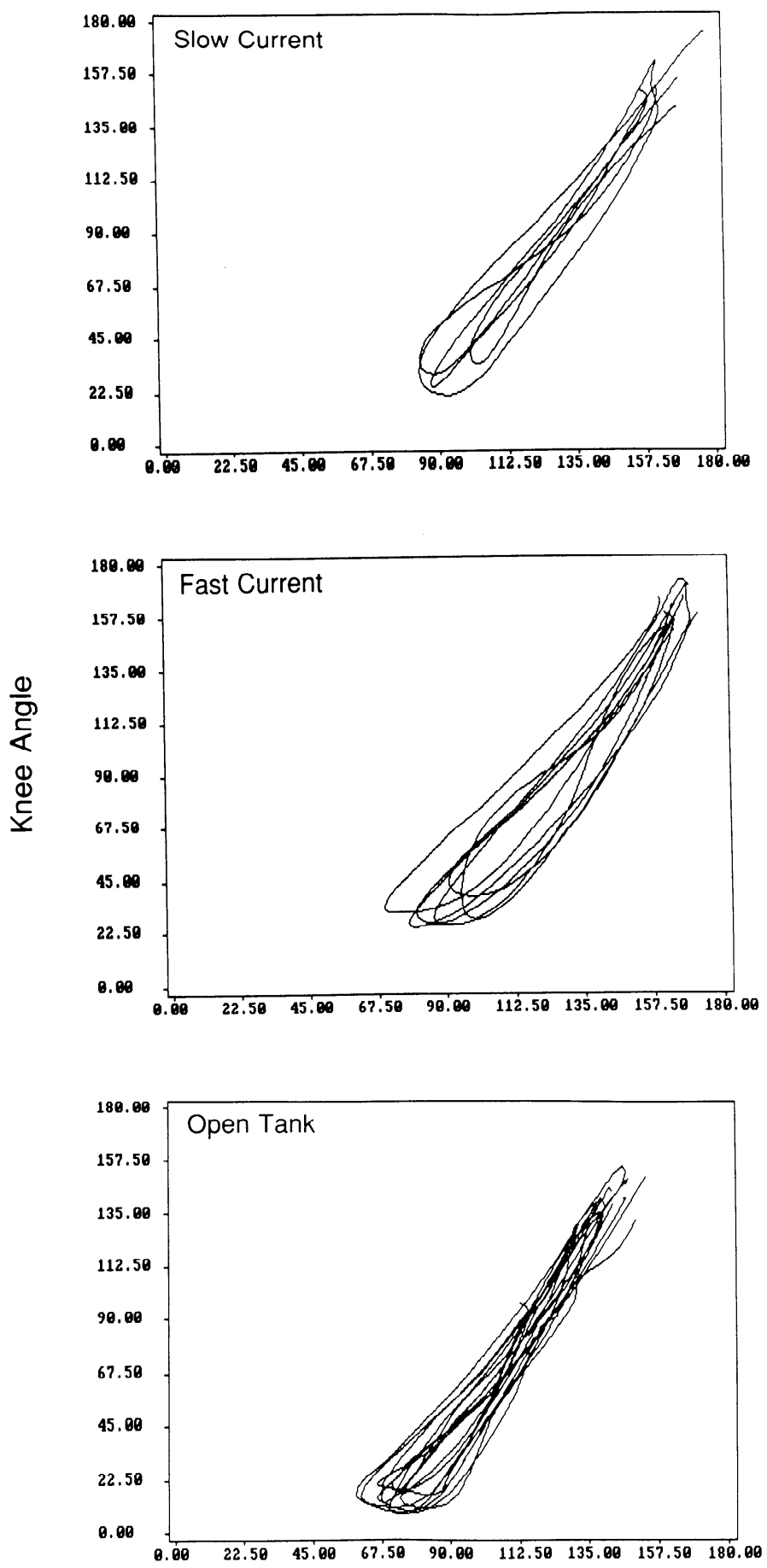

Hip Angle

FIG. 11. Motion paths in hip-knee space for swimming movements in intact $R$. pipiens (top 2 panels) and $R$. temporaria (bottom). Top: out-ofphase interlimb pattern observed at slow current rates. Middle: in-phase pattern seen at fast current speeds. Bottom: paths observed in an open tank. Flexion begins from the top right corner of each panel and proceeds along the bottom portion of the ellipse.
Hip - Knee Velocity Ratio

\section{Swimming}
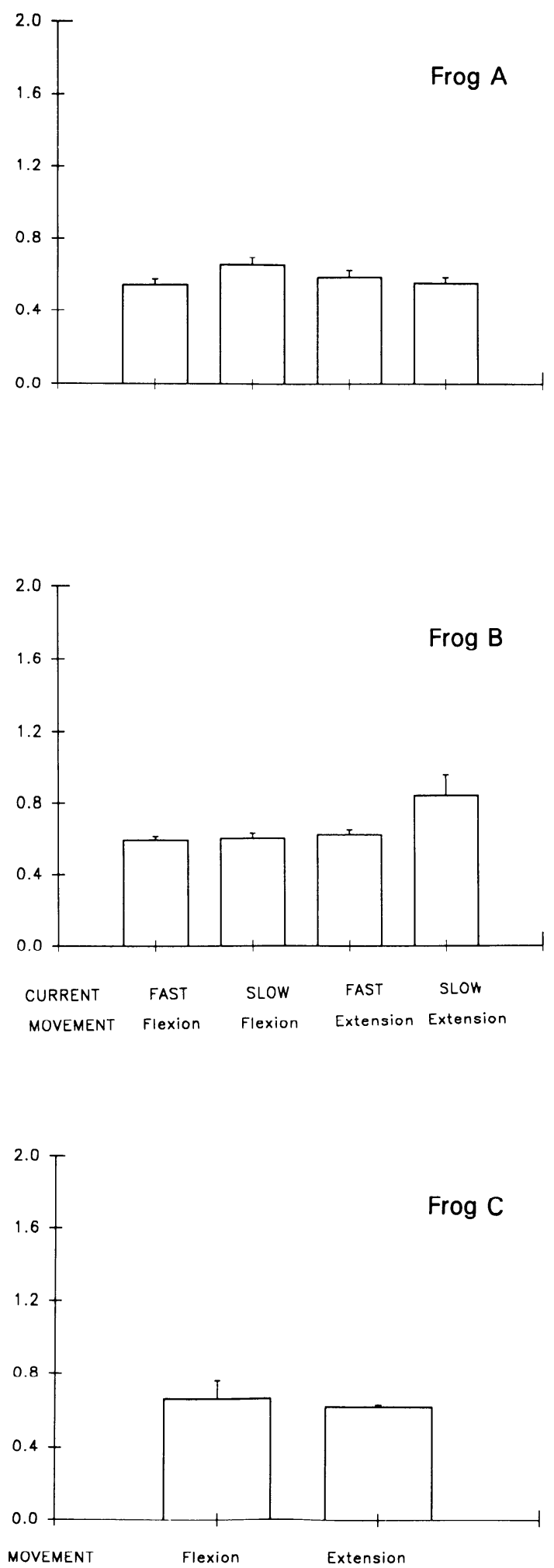

FIG. 12. Ratios of maximum hip velocity to maximum knee velocity in swimming. Frogs $A$ and $B$ were recorded while swimming against imposed currents of different speeds. Frog $C$ was recorded while swimming in an open tank. 
age differences in movement start times were small. Reliable differences in start times were observed in $\sim 10 \%$ of the cases tested $(P<0.01)$; these differences ranged from 2 to $16 \mathrm{~ms}$ with a median value of $8 \mathrm{~ms}$.

In $75 \%$ of the conditions tested, there were no reliable differences in the times at which hip and knee reached maximum velocity. However, reliable differences were observed in two frogs (FR2 and $P H L)$ in three conditions. One of these conditions involves an extreme position in which the knee flexors are greatly stretched (hip 90, knee 135, ankle 135). In this case the average time differences were 45 and 20 ms for $F R 2$ and $P H L$, respectively. The resulting path curvature in this condition is apparent in the top left traces in Fig. 9. A second condition (hip 135, knee 135, ankle 90) involved extreme passive stretch of the ankle extensors, and, in fact, this configuration could only be maintained in $F R 2$ and $P H L$. The observed time differences were 24 and $11 \mathrm{~ms}$, respectively. The final condition (hip 90, knee 90 , ankle 90) did not involve an extreme posture. Time differences between hip and knee maximum velocities of 21 and $16 \mathrm{~ms}$ were observed for $F R 2$ and $P H L$.

Ratios of maximum hip velocity to maximum knee velocity were calculated on a per-trial basis (Fig. 10). ANOVA was used to test for differences among conditions. It can be seen that ratios generally fall within the range $0.35-0.55$, that is, maximum hip velocity typically ranges from 35 to $55 \%$ of maximum knee velocity. The overall ANOVA indi- cated that there were significant differences among ratios. Examination of the figure suggests that the variability can be largely attributed to one or two conditions in which the ratio was higher (e.g., hip, knee, and ankle angles of 135, 90, and $90^{\circ}$ ). Post-hoc pairwise comparisons (Tukey test) showed that only conditions involving the combination of hip and knee angles of 135 and $90^{\circ}$ differed from the others. Otherwise, the ratios were similar in value. The exceptions suggest that the possibility exists for the system to alter the coordination between the joints.

To summarize, joint velocity ratios were similar in the majority of cases in spite of differences in initial joint configuration. Parallel straight line paths were also observed. This is consistent with the view that the nervous system specifies a single direction of equilibrium shift that is the same regardless of initial joint angles.

The data on swimming movements suggest that the direction of equilibrium shift is similar to that observed in withdrawal. Figure 11 shows the paths in hip-knec space for several movements recorded during swimming against slow and fast currents and in an open tank. Hip angle is shown on the horizontal axis. The paths for both flexion and extension movements are approximately straight and have similar slopes regardless of current speed and, consequently, the pattern of interlimb coordination. In the fast current and open tank swimming, hip flexion appears to continue after knee flexion stops (bottom). The angle at
Hip

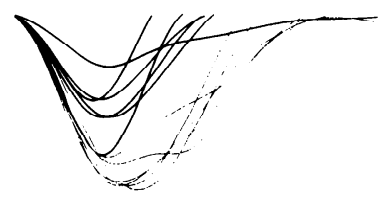

Knee 90 Ankle 90

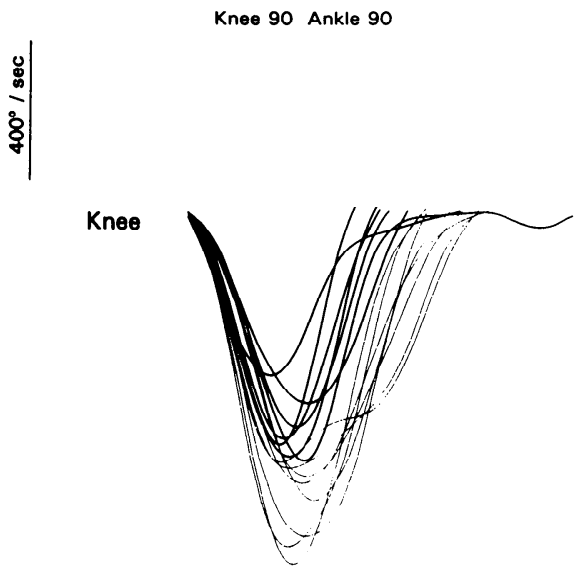

Hip 90 Ankle 90

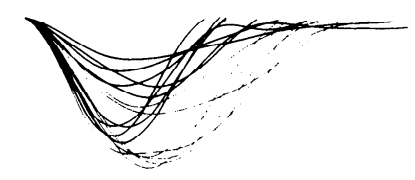

Knee 90 Ankle 135

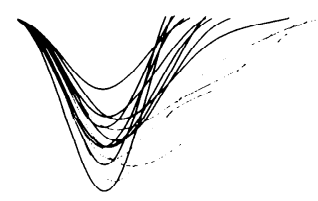

Knee 135 Ankle 90

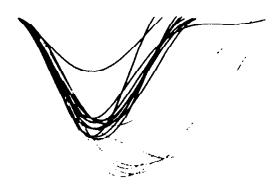

Knee 135 Ankle 135

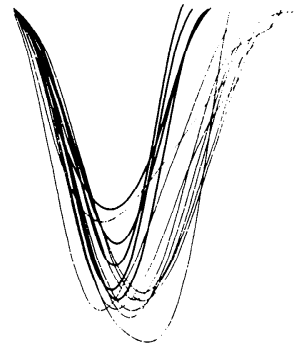

Hip 90 Ankle 135

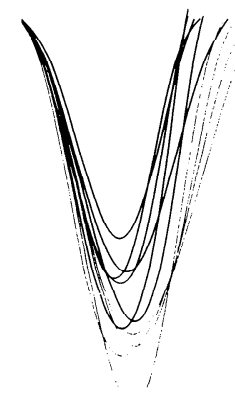

Hip 135 Ankle 90

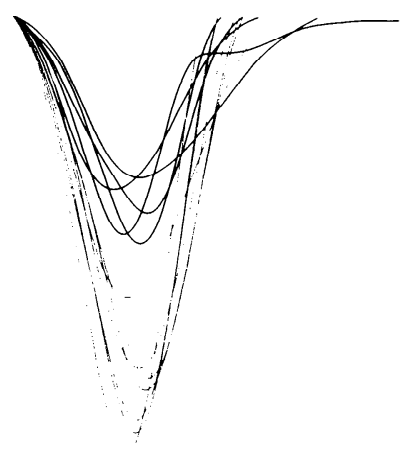

Hip 135 Ankle 135

FIG. 13. Joint angular velocity profiles in withdrawal in spinal $R$. catesbeiana. Top: hip velocity profiles aligned at movement start. Initial hip angles are $90(-)$ or $135^{\circ}(\cdots)$. Corresponding angles at the knee and ankle are shown. Bottom: knee velocity profiles with initial knee angles of $90(-)$ and $135^{\circ}(\cdots)$. Corresponding hip and ankle angles are indicated. 
which the knee stops flexing is similar to that observed in withdrawal. Thus the limiting range of the knee may be achieved in swimming as well.

Ratios of maximum hip velocity to maximum knee velocity are presented in Fig. 12. It can be seen that the ratios fall in the same range $(0.55-0.65)$ as those in withdrawal. The ratios were not found to vary statistically with differences in the current rate or movement direction (flexion vs. extension). Thus, in both withdrawal and swimming, the ratios are invariant across movement conditions.

\section{Joint velocity profiles}

Angular velocity profiles of each joint were examined for different movement amplitudes. Thus, for example, the velocity profiles for large- and small-amplitude hip movements were directly compared. In addition, the velocity profiles of the hip and knee (associated with the same movement trials) were contrasted.

According to the EP hypothesis ( $\lambda$ model), if the rate of shift of the equilibrium position is constant, then motions of different amplitude will have velocity profiles that coincide over an initial phase of the movement and then diverge (see Feldman et al. 1990). Alternatively, if rates of equilib- rium shift are different for movements of different amplitude, then the corresponding velocity profiles will diverge from movement onset. For example, if the rate of shift increases with amplitude, then the initial slope of the velocity profiles will be greater for large-amplitude motions.

Figure 13 shows both hip (top) and knee (bottom) velocity profiles for a single frog $(P H L)$ in all of the conditions tested for withdrawal. The solid curves are the velocity profiles associated with movements from an initial joint angle of $90^{\circ}$. The dotted curves are for an initial angle of $135^{\circ}$. In general, maximum velocities for the knee are greater than those for the hip. In addition, it can be seen that the initial portions of the trajectories are similar for hip movements of different amplitudes. The same is observed for the knee motions with a single exception (hip 135, ankle 135). This is consistent with the idea that the rate of equilibrium shift (corresponding to individual joints) is constant regardless of the initial configuration of the limb. However, the constant rate of shift of the hip may differ from the constant rate of the knee.

Figure 14 presents velocity profiles at the contralateral hip during the crossed-extension reflex. The curves for both large- and small-amplitude movements are shown. The amplitude was manipulated by placing the contralateral

\section{Crossed Extension}

\section{Hip Velocity}

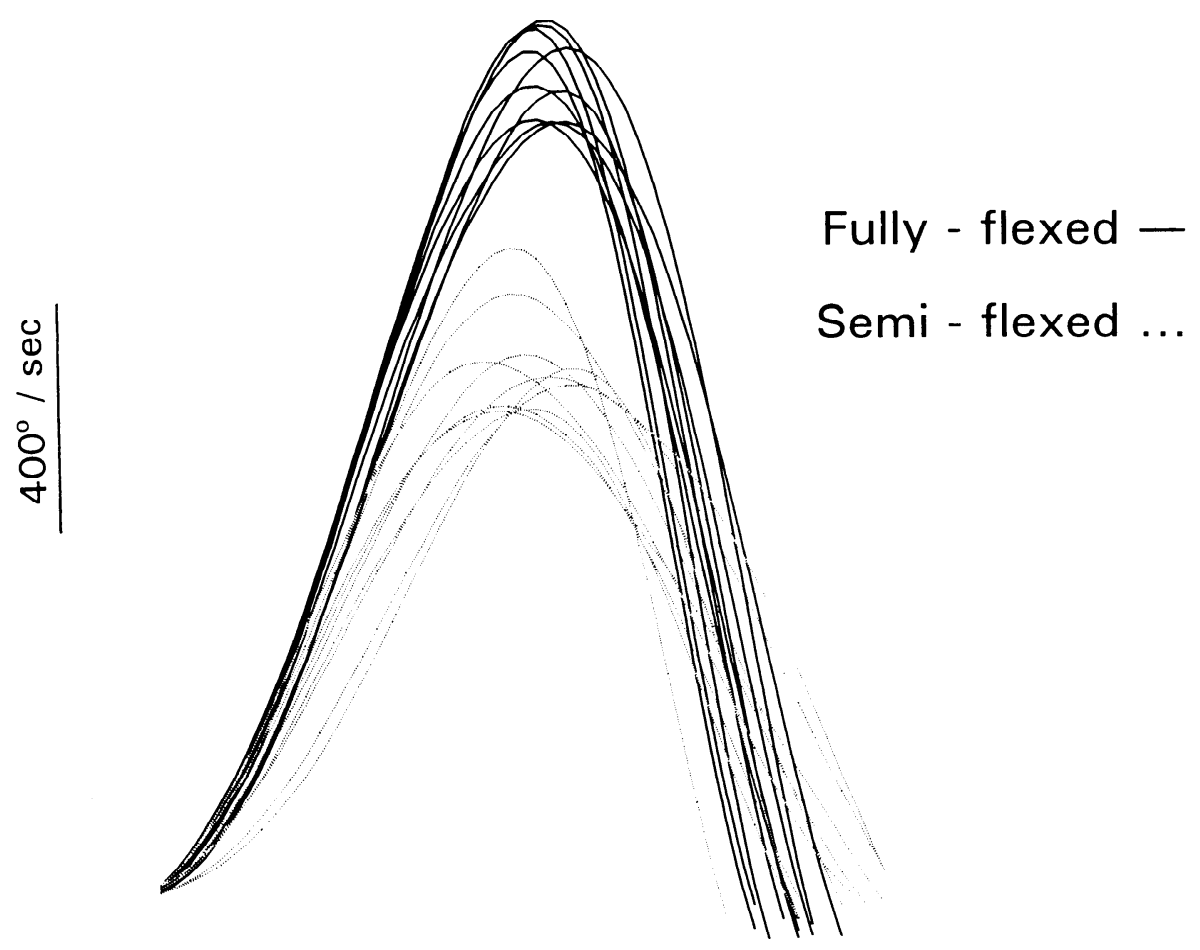

FIG. 14. Hip velocity profiles in crossed-extension reflex with contralateral limb initially semiflexed $(\cdots)$ or fully flexed ( - ). 

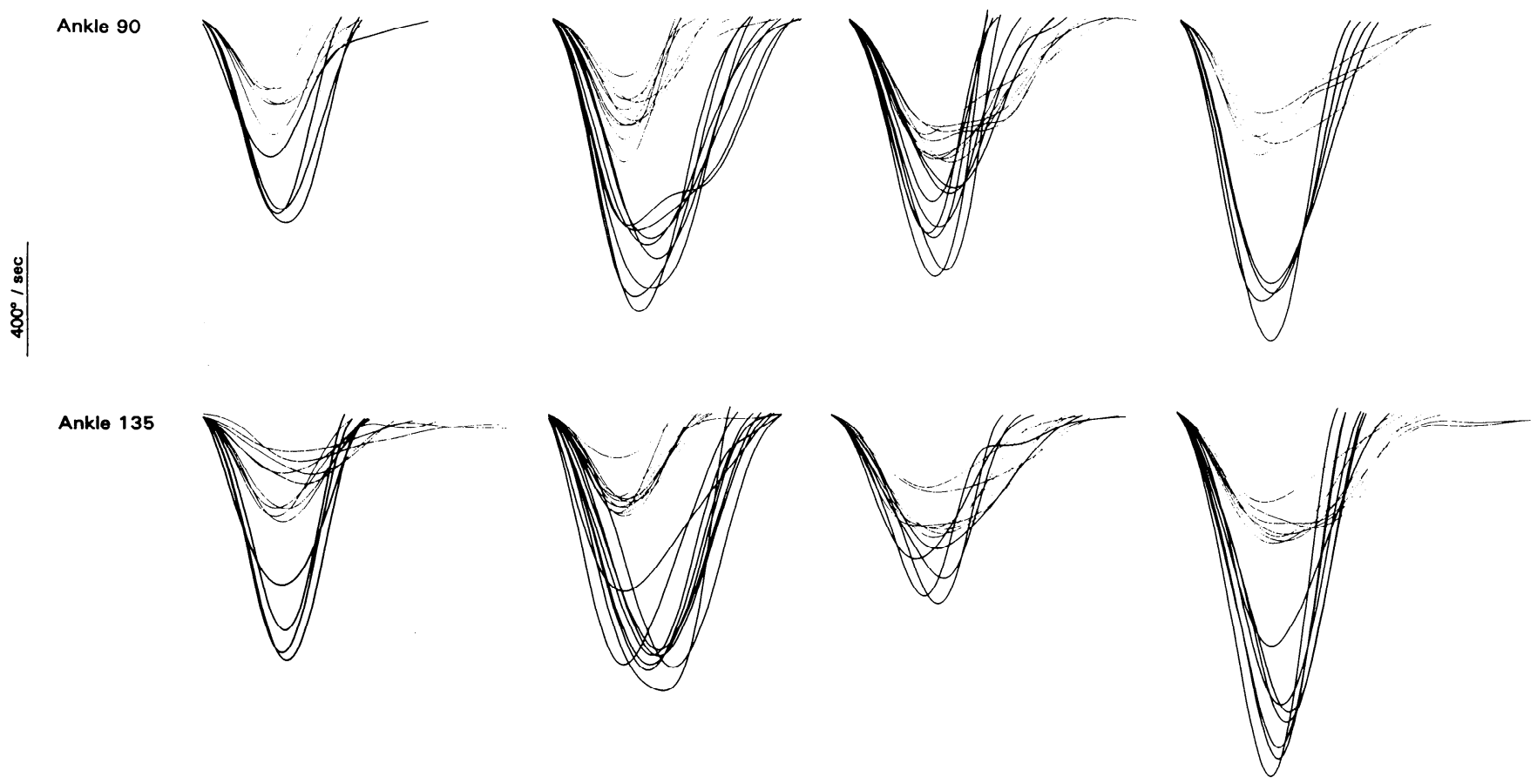

Hip 90 Knee 90

Hip 90 Knee 135

Hip 135 Knee 90

Hip 135 Knee 135

$100 \mathrm{msec}$

FIG. 15. Joint angular velocity profiles of the hip and knee in the withdrawal reflex in the spinal frog. Velocity profiles for the hip $(\cdots)$ and knee $(-)$ are shown for a single frog in all conditions tested.

limb in either an initially semiflexed or an initially fully flexed position. As in withdrawal, the initial portion of the velocity function is similar for movements of different amplitudes. This then also supports the notion that the equilibrium joint positions are shifted at a constant rate regardless of initial joint angle and movement amplitude.

If straight line paths are to be observed in joint space, then joint velocity profiles must be similar in shape and must start and end at the same time. In hindlimb withdrawal the similarity is restricted to the initial portion of the movement before the limiting range of the joint. Figure 15 shows velocity profiles at the hip $(\cdots)$ and the knee $(-)$ for all eight initial joint configurations. The profiles have been lined up at movement start. This is justificd because differences in joint motion onset times were found to be unreliable and small. When the initial angles at the hip and knee are both $90^{\circ}$, the movements tend to reach maximum velocity simultaneously and end at the same time as well. If movements in these conditions were scaled on movement amplitude, similar velocity profiles would be obtained. When initial angles at the hip and knee are 90 and $135^{\circ}$, respectively (one of the extreme initial configurations described above), the hip reaches maximum velocity before the knee and also stops moving sooner. The curvature in the joint space paths in this condition is consistent with this asynchrony in reaching maximum velocity. In the remaining conditions it can be seen that the profiles appear similar in form over the initial portion of the movement and reach maximum velocity simultaneously. In these cases, knee movement stops before hip movement, and the deceleration of the hip is rather slow in comparison to the knee. This results in the characteristic curvature of those hip-knee paths in Fig. 5 in which the initial hip angle is $135^{\circ}$.

In summary, the joint velocity profiles at the hip and knee in withdrawal appear similar up until maximum velocity and, with two exceptions, reach maximum velocity simultaneously. This supports the finding that joint space paths are straight up until this point.

\section{DISCUSSION}

The determinants of the motion path of the hindlimb were explored in both intact and spinal frogs. Hindlimb organization in the spinal preparations was studied by examining the kinematic properties of withdrawal and crossed-extension reflexes elicited from different initial joint configurations. In the intact preparation, the kinematics of swimming were examined.

Three alternative accounts of hindlimb motion were considered. One possibility is that all movements have equilibrium trajectories that lie along a single line in joint space (see Fig. 1). Some evidence for this possibility was provided by preliminary observations that hindlimb paths in swimming tend to converge to a common path. A second possibility is that the direction of the equilibrium trajectory toward a final joint configuration is specified. However, straight line nonparallel paths in joint space, which would provide evidence for this alternative, were not observed. A third alternative is that all equilibrium trajectories share a com- 
mon direction irrespective of initial joint angles. Evidence for this possibility was provided by the observation of parallel paths in joint space with the exception of regions near functional joint limits.

The hindlimb withdrawal reflex was characterized by joint space paths that were initially straight. Note that straight line paths are obtained when the joints move over the same time period and have similar velocity profiles. In addition, path direction was generally independent of initial joint configuration such that parallel paths were observed.

A necessary condition for parallel paths is that the ratio of maximum joint velocities remains constant across movement conditions. This ratio thus provides a simple quantitative measure, which can be used to test the third alternative described above. It was found that these ratios were similar in magnitude over differences in initial angle. Therefore these findings are consistent with the observation of parallel paths in joint coordinates and suggest that the motor system specifies a single direction for equilibrium trajectories. Similar patterns were obtained in swimming movements in the intact frog. Ratios of joint angular velocities were independent of current speed and similar in magnitude to those observed in withdrawal.

Parallel straight line paths in joint coordinates were observed except when joint limits were approached toward the end of the movement. When limits were approached, the paths deviated substantially. Depending on initial joint configuration, the time at which joint limits are encountered will vary. The joints that start from smaller initial angles reach their functional limits earlier and stop first. Thus there is no strict sequence to the order in which individual joint motions end in withdrawal. This pattern was similar for spinal and intact frogs. Consistent with this account is the finding that the amplitude and duration of individual joint movements depend only on the initial angle at that joint, regardless of the initial angles of the other two. Because joint limits do not vary, it is clear that joint amplitude will be affected by initial joint angle. Thus the finding that amplitude depends only on initial joint angle does not reflect a neural control strategy but rather mechanical effects due to the limiting range of the joint.

The EP hypothesis suggests that, in movements without specific constraints, equilibrium joint angles shift at a constant velocity. However, the actual joint velocity profile is smooth (gradually increasing and then decreasing) as a result of neuromuscular and mechanical dynamic effects. Straight lines in joint equilibrium space result when equilibrium joint angles begin to shift simultaneously and each shifts at a constant velocity. All such lines will be parallel if the ratio of shift velocities is preserved across initial joint configurations. We assume that, under these conditions, the actual paths in joint space will be approximately straight.

Velocity profiles at a given joint were similar over the initial part of the movement for movements of different amplitude. This result suggests that the rate of shift of each joint equilibrium angle is constant across initial angles. Consequently, there appears to be a single rate of shift of equilibrium specificd for each joint subserving withdrawal reflexes. Likewise, the rates of shift for the knee and hip in each phase of swimming appear to be constant. Note that, if the rate of equilibrium shift is constant, movement amplitude depends only on the duration of the shift.

Joint velocity functions of the knee and hip were similar in form in conditions where joints did not approach their functional limits. Similar results have been obtained in studies of human arm movement but have been interpreted in different ways. Atkeson and Hollerbach (1985) adopt a computational or robotics point of view. They argue that movements are planned in terms of kinematic trajectories in hand space. The inverse dynamics are then computed to determine the joint torques needed to achieve these trajectories. It was suggested that the scaling of the form of the velocity function with time and load simplifies the computation of the dynamics (Atkeson and Hollerbach 1985; Hollerbach and Flash 1982). In contrast, we believe that movements are controlled by specifying the direction and rate of shift in equilibrium position. Scaling with amplitude and duration results from changes in the rate and duration of the control signal in conjunction with specific neuromuscular and mechanical dynamics (Feldman 1986; Feldman et al. 1990).

In both reflex and nonreflex behaviors, we have observed straight line paths in joint space except near work-space boundaries. This is consistent with the idea that these movements are organized and controlled in joint-level equilibrium coordinates. Straight lines in joint space have been found to approximate a minimum-time solution to trajectory performance (Sahar and Hollerbach 1986). Control strategies that tend to reduce motion duration may well correspond to the needs of the withdrawal response of the frog hindlimb.

In the hindlimb wiping reflex, movements are produced in response to extcrnal stimuli. One may presume that the stimulus location is represented in some space (e.g., 2-dimensional skin coordinates or 3-dimensional Cartesian coordinates) other than joint angle coordinates and that movement generation involves a sensorimotor transformation between stimulus coordinates and joint coordinates. Thus, at some level, wiping movements must be represented in some extrinsic coordinate system, and regularities in this coordinate system may be expected to be observed. In contrast, it is not clear that in withdrawal, crossed extension, and swimming movement, coordinate transformations are required because the movement target is not externally represented. It may be that these movements are planned and organized dircctly at the joint level.

In summary, we have observed parallel straight line motion paths in the withdrawal reflex in the spinal frog in spite of differences in initial joint configuration. In swimming movements in the intact frog, paths of similar slope were observed. This suggests that the direction of the shift of the equilibrium position (in joint coordinates) specified by the nervous system is the same regardless of initial joint angles in withdrawal and that the same direction is preferred in swimming, which requires supraspinal input. Moreover, in withdrawal, crossed extension, and each phase of swimming, the equilibrium position is assumed to be shifted at a constant velocity. This is consistent with the demonstration of similar trajectories over the initial portion of reflex movements of different amplitudes. Overall, the findings suggest that common constructive elements underlie hindlimb movements in both the spinal and intact frog. 
We thank S. Adamovich, M. Berkinblit, O. Fukson, A. Karpovich, T. Tkach, and L. Sergio.

This work was supported by research grants from the Natural Sciences and Engineering Research Council of Canada and the G. W. Stairs Memorial Fund

Address for reprint requests: D. J. Ostry, Dept. of Psychology, McGill University, 1205 Dr. Penfield Ave., Montreal, Quebec H3A 1B1, Canada.

Received 26 March 1990; accepted in final form 5 November 1990.

\section{REFERENCES}

AtKeson, C. G. AND Hollerbach, J. M. Kinematic features of unrestrained vertical arm movements. J. Neurosci. 5: 2318-2330, 1985.

Berkinblit, M. B., FeldMAN, A. G., AND Fukson, O. I. Adaptability of innate motor patterns and motor control mechanisms. Behav. Brain Sci. 9: 585-638, 1986.

DAHLQUIST, G. AND BJÖRCK, Å. Numerical Methods. Englewood Cliffs, NJ: Prentice-Hall, 1969.

Feldman, A. G. Once more for the equilibrium-point hypothesis for motor control. J. Mot. Behav. 18: 17-54, 1986.

Feldman, A. G., Adamovich, S. V., Ostry, D. J., AND Flanagan, J. R.
The origin of electromyograms-explanations based on the equilibrium point hypothesis. In: Multiple Muscle Systems: Biomechanics and Movement Organization, edited by J. Winters and S. Woo. London: SpringerVerlag, 1990.

Flanagan, J. R., Feldman, A. G., AND OSTRY, D. J. The equilibrium point model for two joint arm movement control. Soc. Neurosci. Abstr. 15: 173, 1989.

Giszter, S. F., McInTyre, J., AND BizzI, E. Kinematic strategies and sensorimotor transformations in the wiping movements of frogs. J. Neurophysiol. 62: 750-767, 1989.

Hollerbach, J. M., and Flash, T. Dynamic interactions between limb segments during planar arm movement. Biol. Cybern. 44: 67-77, 1982.

Mortin, L. I., KeIFER, J., AND STEIN, P. S. G. Three forms of scratch reflex in the spinal turtle: movement analysis. J. Neurophysiol. 53 1501-1516, 1985.

OHR, E. A. Tricaine methanesulfonate. I. $\mathrm{pH}$ and its effects on anesthetic potency. Comp. Biochem. Physiol. C Comp. Physiol. 54C: 13-17, 1976.

SAHAR, G. AND HOLlERBACH, J. M. Planning of minimum-time trajectories for robot arms. Int. J. Robotics Res. 5: 90-100, 1986.

Schotland, J. L., LeE, W. A., AND Rymer, W. Z. Wipe and flexion withdrawal reflexes display different EMG patterns prior to movement onset in the spinalized frog. Exp. Brain Res. 78: 649-653, 1989. 